\title{
SEIS IDEAS QUE ESTÁN CAMBIANDO EL CAMPO DE LAS DISCAPACIDADES INTELECTUALES Y DEL DESARROLLO EN TODO EL MUNDO
}

\section{Six ideas that are changing the IDD field internationally}

Robert L. SCHALOCK, Ph.D. Professor Emeritus, Hastings College (Nebraska-USA) rschalock@ultraplix.com

Recepción: 6 de noviembre de 2017

Aceptación definitiva: 7 de noviembre de 2017

RESUMEN: El propósito del presente artículo es presentar seis ideas que en estos momentos están teniendo influencia en los legisladores y en los proveedores de servicios y apoyos en el ámbito de las discapacidades intelectuales y del desarrollo (que están íntimamente relacionadas). Estas seis ideas son las siguientes: las personas se ven influidas por varios sistemas, debemos enfocar la discapacidad desde un punto de vista holístico, las políticas sobre discapacidad deben tener un enfoque sistemático, los apoyos deben abarcar los elementos de un sistema de apoyos, la evaluación tiene diversos factores y las organizaciones se deben transformar para poder ser sostenibles.

Palabras Clave: discapacidad intelectual; discapacidad del desarrollo; apoyos; transformación organizacional; sistemas; política social.

AвSTRACT: The purpose of this article is to discuss six ideas that are currently impacting policy makers and service/support providers in the field of intellectual and closely related developmental disabilities. These six ideas are that people are influenced by multiple systems, disability should be approached holistically, disability policy needs to be approached systematically, supports should encompass elements of a system of supports, evaluation is multifaceted, and organizations need to transform to be sustainable. 


\section{SEIS IDEAS QUE ESTÁN CAMBIANDO EL CAMPO DE LAS DISCAPACIDADES INTELECTUALES \\ Y DEL DESARROLLO EN TODO EL MUNDO \\ ROBERT L. SCHALOCK}

KEY WORDS: intellectual disability; developmental disability; supports; organizational transformation; systems; disability policy.

\section{Introducción y descripción general}

L

AS IDEAS SON LOS CIMIENTOS DEL PENSAMIENTO Y DE LA ACCión. En las últimas tres décadas, una serie de ideas y conceptos han modificado la forma en que las sociedades ven a las personas con discapacidad y discapacidades del desarrollo íntimamente relacionadas (DID) y cómo los sistemas de prestación de servicios han respondido a estas ideas y conceptos cambiando los servicios y los apoyos que proporcionan a las personas con DID. Entre ellas, las más destacadas fueron la calidad de vida, la definición de discapacidad intelectual, la estructura de factores del comportamiento adaptativo y el paradigma de los apoyos. Se puede encontrar más información sobre estas ideas, conceptos y cambios en Schalock (2017), Schalock y Verdugo (2013), Turnbull y Stowe (2017) y Verdugo, Navas, Gómez y Schalock (2012).

Hoy en día, los legisladores y los profesionales se ven cuestionados por ideas adicionales que están cambiando el campo de las DID en todo el mundo. El propósito de este artículo es describir esas seis ideas y presentar cómo cada una tiene un efecto en las políticas y las prácticas relacionadas con las DID. Estas seis ideas son las siguientes: (a) las personas se ven influidas por varios sistemas, (b) debemos enfocar la discapacidad desde un punto de vista holístico, (c) las políticas sobre discapacidad deben tener un enfoque sistemático, (d) los apoyos deben abarcar los elementos de un sistema de apoyos, (e) la evaluación tiene diversos factores y (f) las organizaciones se deben transformar para poder ser sostenibles.

\section{Las personas se ven influidas por varios sistemas}

Todos vivimos en sociedades que incluyen a la persona y su familia (es decir, el microsistema); las organizaciones y la propia comunidad (es decir, el mesosistema), y el sistema más amplio, que incluye la cultura propia (es decir, el macrosistema). Estos tres sistemas definen el contexto en el que viven, interactúan, se forman, trabajan y disfrutan las personas. Entender estos sistemas y utilizar el pensamiento sistémico presenta un marco en el que conceptualizar el rol de la asignación de recursos y del desarrollo de oportunidades para mejorar los resultados personales e identificar los factores que influyen en ellos.

\subsection{Asignación de recursos y desarrollo de oportunidades}

Los recursos son mucho más que puro capital financiero. Los recursos también incluyen tiempo, conocimientos, experiencia, capital social tecnológico y colaboraciones. Del mismo modo, el desarrollo de oportunidades implica crear entornos 
que fomenten el crecimiento y el desarrollo, apoyen a las personas y se adapten a las necesidades psicológicas relacionadas con la autonomía, la competencia y los nexos personales.

Como se muestra en la Figura 1, la asignación de recursos y el desarrollo de oportunidades se pueden analizar desde una perspectiva sistémica. Hay que tener en cuenta que el desarrollo de oportunidades fluye del microsistema al macrosistema, mientras que los recursos fluyen del macrosistema al microsistema.

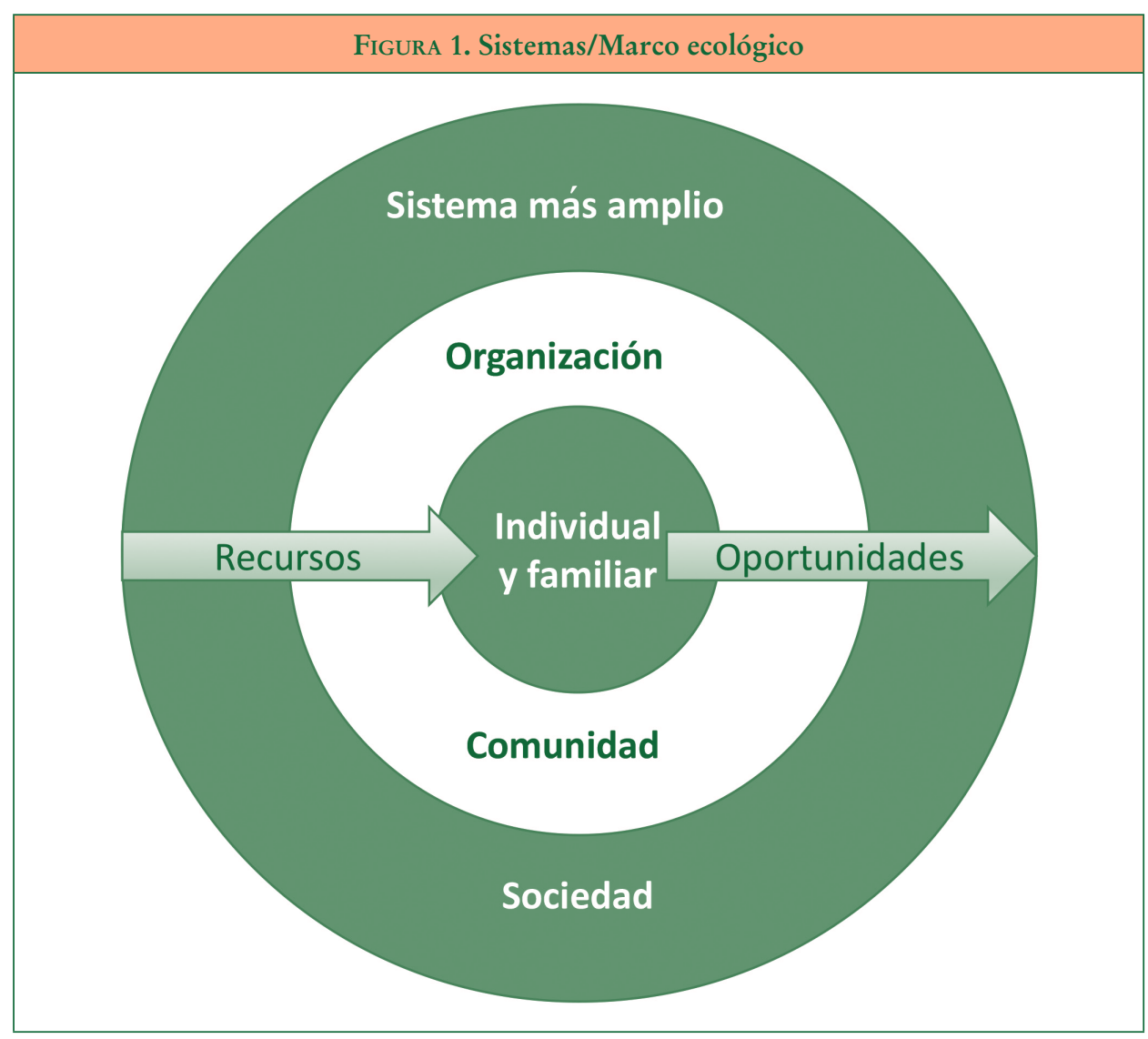

\subsection{Factores que influyen en los resultados personales}

Se puede usar un marco de trabajo con varios sistemas para identificar valores sistémicos que influyen en los resultados personales. La Tabla 1 resume estos factores basándose en el trabajo de Claes et al. (2012), Schalock y Verdugo (2012) y Shogren, Luckasson y Schalock (2015). 


\begin{tabular}{|c|c|}
\hline Nivel de sistemas & Factores de ejemplo \\
\hline Microsistema & $\begin{array}{l}\text { - Objetivos personales y necesidades de apoyo evaluadas } \\
\text { - Puntos fuertes y activos } \\
\text { - Limitaciones funcionales (es decir, funcionamiento intelectual, } \\
\text { comportamiento adaptativo, salud) } \\
\text { - Participación familiar }\end{array}$ \\
\hline Mesosistema & $\begin{array}{l}\text { - Prácticas en la prestación de servicios y apoyos } \\
\text { - Estrategias de apoyos utilizadas (es decir, un sistema de apoyos) } \\
\text { - Entornos estables y predecibles }\end{array}$ \\
\hline Macrosistema & $\begin{array}{l}\text { - Actitudes de la sociedad } \\
\text { - Iniciativas políticas (por ejemplo, empleo con apoyo, vida con apoyo, } \\
\text { educación inclusiva) } \\
\text { - Prestación de apoyos continuada }\end{array}$ \\
\hline
\end{tabular}

\section{El enfoque de la discapacidad debe ser holístico}

En la actualidad existen cuatro perspectivas que ayudan a explicar la discapacidad intelectual (DI). Cada perspectiva representa una visión del mundo particular; explora la repercusión de los diferentes factores que influyen en la DI; ofrece una base para las intervenciones y los apoyos vinculada a esa visión del mundo, y organiza la información pertinente dándole una forma procesable para mejorar su comprensión y para utilizarla como punto de partida para unas mejores recomendaciones y decisiones. Estas cuatro perspectivas utilizan los enfoques biológicos, psicoeducativos, socioculturales o de la justicia. Aunque cada una de estas cuatro perspectivas actualmente cumple importantes objetivos, y seguirá haciéndolo, de forma independiente no explican la complejidad de la DI, ni proporcionan individualmente un marco de trabajo teórico holístico que nos ayude a comprender la DI y nos guíe en nuestros esfuerzos por aliviar su impacto. La perspectiva biomédica se centra en los factores genéticos y psicológicos que producen la DI. La perspectiva psicoeducativa destaca las limitaciones intelectuales, psicológicas o conductuales, y del aprendizaje asociadas con la DI. La perspectiva sociocultural se centra en la interacción entre las personas y sus entornos mediante la cual el significado social de la DI se desarrolla a partir de las creencias, las conductas, el lenguaje y los acontecimientos de la sociedad relacionados con las personas con DI, y en las respuestas de estos individuos ante dicha interacción. La perspectiva de la justicia subraya el hecho de que todas las personas, incluidas aquellas que han recibido un diagnóstico de DI, tienen los mismos derechos humanos y legales.

Hace poco que estas cuatro perspectivas se integraron en un marco de trabajo teórico bolístico que se puede utilizar para explicar la discapacidad intelectual y organizar la información correspondiente en forma de una hoja de ruta procesable que oriente su comprensión y su aplicación. En la Tabla 2 se incluyen los cinco componentes de este marco de trabajo holístico, que se tratan en más detalle en Schalock, Luckasson, Tasse y Verdugo (en prensa). 
TABLA 2. Componentes de un enfoque integrado sobre la discapacidad intelectual

\begin{tabular}{|l|l|}
\hline Componente & Descripción \\
\hline $\begin{array}{l}\text { Explicación de discapacidad } \\
\text { intelectual }\end{array}$ & $\begin{array}{l}\text { La discapacidad intelectual se caracteriza por limitaciones } \\
\text { significativas tanto del funcionamiento intelectual como del } \\
\text { comportamiento adaptativo, que se expresan en las habili- } \\
\text { dades adaptativas conceptuales, sociales y prácticas. La DI se } \\
\text { origina durante el periodo de desarrollo }\end{array}$ \\
\hline $\begin{array}{l}\text { Locus de la discapacidad } \\
\text { intelectual }\end{array}$ & $\begin{array}{l}\text { - Deficiencia genética, neurológica o psicológica; limitacio- } \\
\text { nes significativas en el funcionamiento intelectual y en las } \\
\text { habilidades conceptuales, prácticas o sociales; situaciones } \\
\text { y condiciones del entorno }\end{array}$ \\
\hline $\begin{array}{l}\text { Intervenciones y apoyos para } \\
\text { la prevención o mitigación de } \\
\text { la discapacidad intelectual }\end{array}$ & $\begin{array}{l}\text { - Intervenciones profesionales } \\
- \text { Entornos inclusivos } \\
\text { - Estrategias de apoyos individualizados }\end{array}$ \\
\hline $\begin{array}{l}\text { Clasificación en subgrupos de } \\
\text { las personas con discapacidad } \\
\text { intelectual }\end{array}$ & $\begin{array}{l}\text { Etiología; niveles de desarrollo, funcionamiento intelectual, } \\
\text { comportamiento adaptativo e intensidad de los apoyos nece- } \\
\text { sarios; designaciones legales o reglamentarias (es decir, com- } \\
\text { petencia/incompetencia; apto/no apto) }\end{array}$ \\
\hline
\end{tabular}

\section{Las políticas sobre discapacidad deben tener un enfoque sistemático}

Las políticas sobre discapacidad deben reflejar tres tendencias o avances recientes en este campo (Schalock, 2017). En primer lugar, experimentamos el cambio y la transformación no solo en el ámbito de las DID, sino también en los entornos sociopolíticos en los que las personas con DID y sus familias viven y operan los servicios de prestación de servicios. En segundo lugar, cada vez es más necesario evaluar cómo las políticas sobre discapacidad influyen en las vidas y los resultados valorados de las personas con DID. En tercer lugar, nos hemos dado cuenta de que las políticas sobre discapacidad no son solo acciones de alto nivel de los Gobiernos nacionales, estatales o regionales, sino que abarcan una amplia variedad de enfoques de organizaciones y sistemas que repercuten en las circunstancias sociales, el acceso, las oportunidades educativas, el empleo, la vivienda, las necesidades financieras y la prestación de servicios y apoyos.

Enfrentarse con éxito a estas tendencias y avances exige que aceptemos que la evaluación, la implementación y el desarrollo de políticas sobre discapacidad son procesos dinámicos y que estos procesos se comprenden y se plasman mediante un enfoque sistemático. En la Tabla 3 se resume una visión general de este enfoque y se describe en más detalle en Claes et al. (2017), Schalock (2017), Turnbull y Stowe (2017) y Verdugo et al. (2017). 
TABLA 3. Componentes de un enfoque sistemático de las políticas sobre discapacidad

\begin{tabular}{|l|l|}
\hline Fase de la política & Factores clave \\
\hline Desarrollo & $\begin{array}{l}\text { Basar las políticas en conceptos y principios esenciales como la Con- } \\
\text { vención sobre los derechos de las personas con discapacidad de la } \\
\text { ONU y el concepto de calidad de vida } \\
\text { - Incluir los resultados deseados de las políticas en su desarrollo para } \\
\text { guiar la implementación y la evaluación de las políticas } \\
\text { - Incorporar los conocimientos actuales sobre las DID }\end{array}$ \\
\hline Implementación & $\begin{array}{l}\text { - Realizar análisis contextuales para identificar los factores que blo- } \\
\text { quean o impulsan el cambio }\end{array}$ \\
& $\begin{array}{l}\text { Alinear los objetivos de las políticas con intervenciones, servicios y } \\
\text { apoyos específicos } \\
\text { - Crear ámbitos de colaboración }\end{array}$ \\
\hline Evaluación & $\begin{array}{l}\text { Centrar la evaluación en los cambios personales, familiares y en la } \\
\text { sociedad } \\
\text { - Incorporar un modelo de evaluación } \\
\text { - Identificar y evaluar los indicadores basados en las evidencias } \\
- \text { Usar estándares de evaluación y estrategias de recopilación de eviden- } \\
\text { cias basados en las mejores prácticas }\end{array}$ \\
\hline
\end{tabular}

\section{Los apoyos deben abarcar los elementos de un sistema de apoyos}

El paradigma de los apoyos y la prestación de apoyos individualizados se han convertido en el principal mecanismo de prestación de servicios en la mayor parte del mundo. El paradigma de los apoyos reúne las prácticas procedentes de la planificación centrada en la persona, el desarrollo personal y las oportunidades de crecimiento, la inclusión en la comunidad, la autodeterminación, el empoderamiento y los resultados de evaluación. Y lo consigue haciendo lo siguiente: (a) subrayando que se puede gestionar cualquier asimetría entre persona y entorno que suponga una necesidad de apoyos mediante el uso razonable de estrategias de apoyos individualizados, en lugar de centrarnos en "arreglar a la persona"; (b) dedicando los servicios y los apoyos a superar el vacío entre el "qué es" y "lo que puede ser”, y (c) viendo a las personas con DID según el tipo y la intensidad de los apoyos necesarios, en lugar de sus limitaciones o su diagnóstico.

El concepto de los apoyos y la presentación de apoyos han evolucionado en las últimas dos décadas gracias a nuestra mejor comprensión del concepto de los sistemas de apoyos. Este concepto ampliado se muestra gráficamente en la Figura 2. Como se muestra en la figura, un sistema de apoyos abarca la provisión de intervenciones profesionales, la creación de entornos y la prestación de estrategias de apoyos individualizados. En la Tabla 4 se proporciona un resumen de cada componente. Los componentes y los elementos que se presentan en esta tabla se basan en la síntesis de la literatura actual al respecto presentada por Lombardi Chiu, Schalock y Claes (2017) y Schalock et al. (en prensa). 


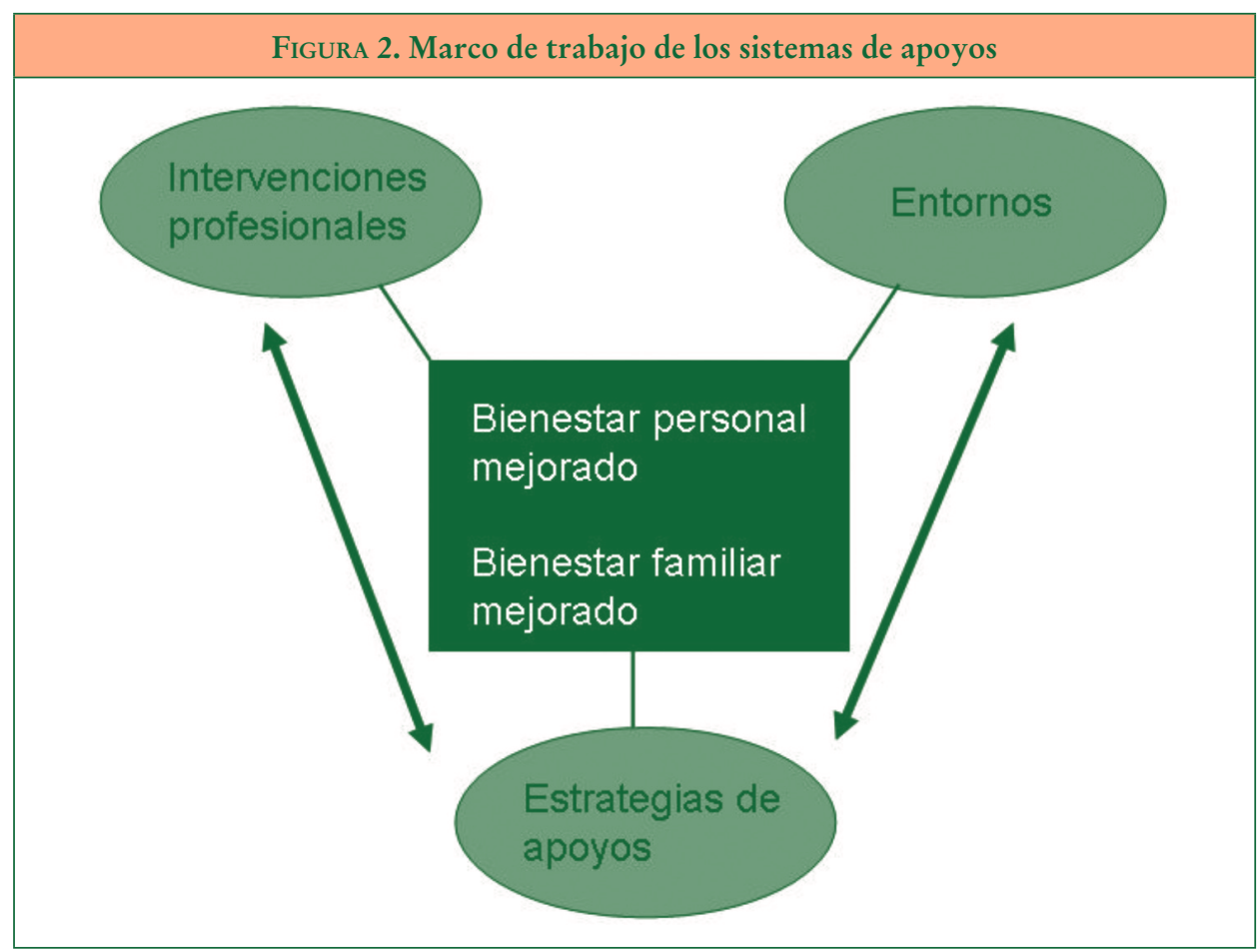

TABLA 4. Componentes y elementos de un sistema de apoyos

\begin{tabular}{|l|l|}
\hline Componente & Elementos \\
\hline Intervenciones profesionales & - Alimentarios o nutricionales \\
& - Médicos o quirúrgicos \\
& - Protésicos \\
& - Formación y desarrollo de crianza, profesionales y \\
& profesores \\
& - Estrategias de apoyos educativos \\
& - Adaptación del entorno \\
& - Empleo \\
& - Participación en la comunidad \\
& - Reforma de políticas \\
& - Afirmación de derechos \\
\hline Entornos inclusivos & - Entornos que (a) fomentan el crecimiento y el desarrollo, \\
& apoyan a las personas, y (b) se adaptan a las necesidades \\
& psicológicas relacionadas con la autonomía, la \\
& competencia y los nexos personales \\
& - Ejemplos: empleo con apoyo, vida con apoyo, educación \\
& inclusiva y envejecimiento en el hogar \\
\hline
\end{tabular}

(C) Ediciones Universidad de Salamanca / CC BY-NC-ND

Siglo Cero, vol. 49 (1), n. ${ }^{\circ}$ 265, 2018, enero-marzo, pp. 7-19 
TABLA 4. Componentes y elementos de un sistema de apoyos (cont.)

\begin{tabular}{|l|l|}
\hline Componente & Elementos \\
\hline Estrategias de apoyos & - Apoyos naturales \\
individualizados & - Tecnología \\
& - Protésicos \\
& - Educación a lo largo de la vida \\
& - Adaptación razonable \\
& - Dignidad y respeto \\
& - Puntos fuertes y activos personales \\
\hline
\end{tabular}

\section{La evaluación tiene múltiples facetas}

La evaluación implica una actividad explícita y planificada cuyo propósito es la determinación del efecto de las políticas y las prácticas relacionadas con las DID. Como se muestra en la Tabla 5, la evaluación relacionada con las DID se puede concentrar en los resultados personales, en los resultados de la organización, en los resultados de políticas públicas o en los valores sociales. Cada uno de estos aspectos implica a diferentes agentes, tiene una perspectiva sobre la evidencia e indicadores de evidencia consensuados.

\begin{tabular}{|l|l|l|l|}
\hline \multicolumn{3}{|c|}{ TAbla 5. Matriz de evaluación basada en evidencias } \\
\hline $\begin{array}{l}\text { Foco de la } \\
\text { evaluación }\end{array}$ & $\begin{array}{c}\text { Principales partes } \\
\text { interesadas }\end{array}$ & $\begin{array}{c}\text { Perspectiva sobre } \\
\text { la evidencia }\end{array}$ & \multicolumn{1}{c|}{$\begin{array}{c}\text { Indicadores } \\
\text { de evidencia* }\end{array}$} \\
\hline $\begin{array}{l}\text { Resultados } \\
\text { personales }\end{array}$ & $\begin{array}{l}\text { Personas } \\
\text { Familias }\end{array}$ & $\begin{array}{l}\text { Fenomenológica- } \\
\text { existencial }\end{array}$ & $\begin{array}{l}\text { - Persona: puntuaciones per- } \\
\text { sonales en las dimensiones de } \\
\text { calidad de vida; mediciones de } \\
\text { funcionamiento humano } \\
-\begin{array}{l}\text { Familia: puntuaciones perso- } \\
\text { nales en las dimensiones de } \\
\text { calidad de vida; mediciones de } \\
\text { unidad e integración familiar }\end{array} \\
-\begin{array}{l}\text { Mediciones de posición socio- } \\
\text { económica (educación, salud, } \\
\text { ocupación) }\end{array}\end{array}$ \\
\hline $\begin{array}{l}\text { Resultados } \\
\text { de la } \\
\text { organización } \\
\text { (outputs) }\end{array}$ & $\begin{array}{l}\text { Gestores de } \\
\text { programas } \\
\text { Consejo de dirección } \\
\text { Entidades regulatorias } \\
\text { o financiadoras }\end{array}$ & rendimiento & $\begin{array}{l}\text { - Indicadores de eficacia (resul- } \\
\text { tados personales; opciones del } \\
\text { programa) } \\
\text { Indicadores de eficiencia } \\
\text { (costes unitarios, tasas de gas- } \\
\text { toserativos, porcentaje de } \\
\text { presupuesto asignado a los } \\
\text { apoyos directos, alineación } \\
\text { vertical y horizontal) }\end{array}$ \\
\hline
\end{tabular}




\section{TABLA 5. Matriz de evaluación basada en evidencias (cont.)}

\begin{tabular}{|l|l|l|l|}
\hline $\begin{array}{c}\text { Foco de la } \\
\text { evaluación }\end{array}$ & \multicolumn{1}{|c|}{$\begin{array}{c}\text { Principales partes } \\
\text { interesadas }\end{array}$} & $\begin{array}{c}\text { Perspectiva sobre } \\
\text { la evidencia }\end{array}$ & \multicolumn{1}{c|}{$\begin{array}{c}\text { Indicadores } \\
\text { de evidencia* }\end{array}$} \\
\hline $\begin{array}{l}\text { Resultados } \\
\text { de políticas } \\
\text { públicas }\end{array}$ & $\begin{array}{l}\text { Legisladores } \\
\text { Receptores del } \\
\text { programa } \\
\text { Sociedad }\end{array}$ & Postestructural & $\begin{array}{l}\text { - Estatus educativo } \\
\text { - Estatus habitacional } \\
\text { - Estatus ocupacional } \\
\text { - Estado de salud }\end{array}$ \\
\hline Valor social*** & $\begin{array}{l}\text { Analistas de políticas } \\
\text { sociales } \\
\text { Legisladores } \\
\text { Financiadores } \\
\text { o financiación } \\
\text { gubernamental }\end{array}$ & Empírica-analítica & $\begin{array}{l}\text { - Cálculos de costes } \\
\text { - Índices de beneficios } \\
\text { - Ratios coste/beneficio } \\
\text { - Índices de retorno social de la } \\
\text { inversión (SROI, en inglés) }\end{array}$ \\
\hline
\end{tabular}

* Los estándares de evaluación y las estrategias de recopilación de evidencias basados en las mejores prácticas se tratan en SCHALOCK et al. (2017) y SCHALOCK et al. (2011).

** El valor social, los análisis coste-beneficio y el retorno social de la inversión se tratan en Nicholis (2017) y YATES Y MARRA (2017).

Aunque este artículo no se centra en la explicación de la Tabla 5 en detalle, la tabla resume dos aspectos que están especialmente relacionados con esta quinta idea, un concepto que no solo está cambiando el campo de las DID en todo el mundo, sino que también está teniendo un efecto directo sobre los legisladores y sobre los proveedores de servicios: las diferentes perspectivas sobre la evidencia y los indicadores de evidencia que se usan para evaluar los resultados personales.

\subsection{Perspectivas sobre la evidencia}

La evaluación requiere evidencia. La evidencia se define como el conjunto de hechos o información disponible que indica si la creencia o la propuesta es verdadera o válida, y se usa para adoptar decisiones informadas, y se puede analizar desde diferentes perspectivas. Como se describe de forma más exhaustiva en Schalock et al. (2017) y Schalock, Verdugo y Lee (2016), en la actualidad hay cuatro perspectivas sobre la evidencia:

- Empírica-analítica: se centra en los resultados experimentales o científicos que se obtienen a partir de las estrategias de recopilación de datos, incluidas las evidencias aleatorias, los diseños experimentales o controlados, los diseños cuasiexperimentales, los diseños con varias líneas base o los diseños multivariante.

- Fenomenológica-existencial: se centra en las experiencias informadas y en el funcionamiento humano mejorado, la participación social y el bienestar personal, con estrategias de recopilación de datos asociadas, incluidos los autoinformes, los estudios de caso, las etnografías, las investigaciones de acción participativa, los diseños multivariante o el muestreo teórico. 
- Postestructural: se centra en los resultados de políticas públicas deseados, que se evalúan empleando un conjunto de diseños de métodos, diseños multivariante, estudios poblacionales, metaanálisis y registros de datos.

- Gestión del rendimiento: se centra en información o hechos confirmados empíricamente que pueden ser utilizados por las organizaciones para la creación de informes, la supervisión, la evaluación, la investigación y la mejora continua de la calidad. Las estrategias de recopilación de datos incluyen la evaluación de resultados, la auditoría de resultados, las autoencuestas, los análisis de modelos lógicos de programas y las revisiones externas.

\subsection{Indicadores de la evidencia}

En la evaluación de los resultados personales, los indicadores de evidencia más comunes en relación con las DID son las puntuaciones en las dimensiones de calidad de vida de la persona, los índices de funcionamiento humano relacionados con el comportamiento adaptativo, el funcionamiento intelectual, la participación y la salud, las puntuaciones en las dimensiones de calidad de vida de la familia y las mediciones de integración y unidad de la familia (Claes et al., 2017). Independientemente de los indicadores seleccionados, si se satisfacen los siguientes criterios se garantizarán la calidad, la solidez y la pertinencia de las evidencias obtenidas: (a) la evaluación se basa en un modelo conceptual correctamente formulado y validado; (b) se usan indicadores que incluyen los hechos culturales; (c) los indicadores se definen operativamente y se miden con fiabilidad y validez, y (d) se utilizan procedimientos de puntuación estandarizados (Gómez y Verdugo, 2016; Schalock y Keith, 2016).

\section{Las organizaciones se deben transformar para poder ser sostenibles}

Las organizaciones que trabajan con las DID se están transformando para adaptarse a los retos de hoy en día y para llegar a ser más eficaces, eficientes y sostenibles (Reinders, 2008; Schalock y Verdugo, 2013; Schalock et al., 2016). Recientemente, Schalock, Verdugo y van Loon (enviado para su publicación) han desarrollado un modelo basado en la literatura científica y en la experiencia práctica que sirve para orientar la transformación futura de las organizaciones y de los sistemas. Este modelo se presenta en la Figura 3.

Hay tres aspectos de estos modelos que están directamente relacionados con este artículo. En primer lugar, dos de los componentes del modelo se basan en el contexto (los pilares de transformación y las estrategias de transformación) y dos están relacionados con la sostenibilidad (capacidad de la organización y outputs y resultados organizativos). Segundo, la sostenibilidad de la organización está directamente relacionada con su capacidad y aptitud para evaluar los resultados personales y los outputs de la organización (véase la Tabla 5 para obtener información específica sobre estos dos niveles de evaluación). En tercer lugar, una característica exclusiva del modelo: las estrategias de 


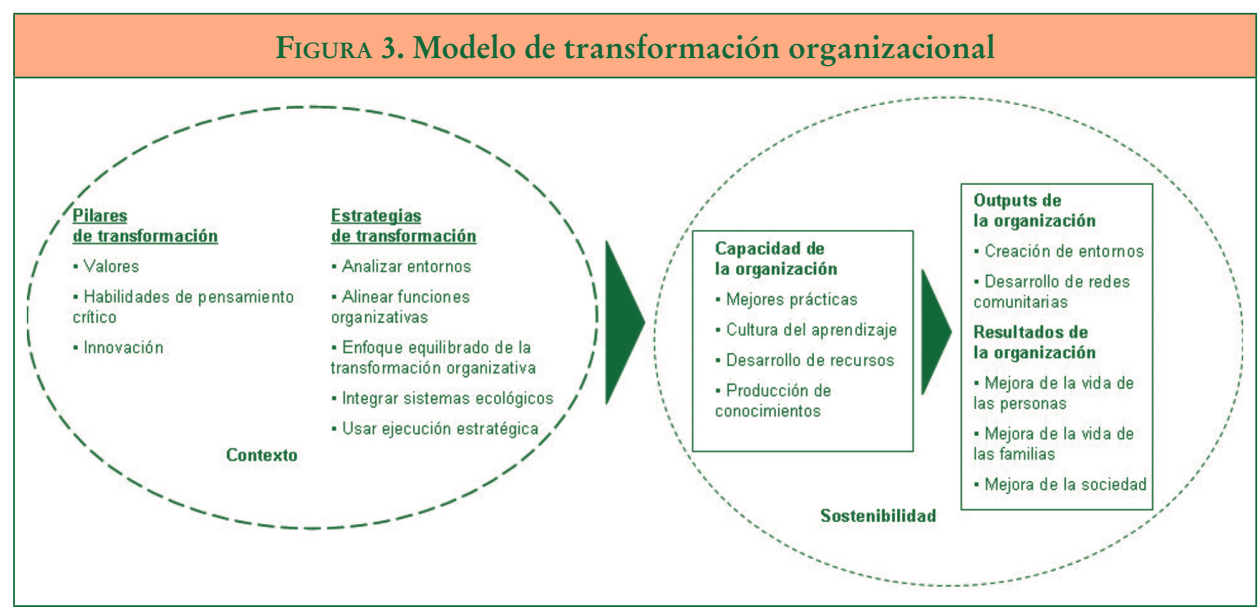

transformación se pueden alinear con respecto a las habilidades de pensamiento crítico necesarias para la transformación organizativa. Este alineamiento se resume en la Tabla 6 y se describe más ampliamente en Schalock et al. (enviado para su publicación).

\begin{tabular}{|l|l|}
\hline \multicolumn{2}{|c|}{ TABLA 6. Alineación de las habilidades de pensamiento crítico y las estrategias } \\
de transformación
\end{tabular}

(C) Ediciones Universidad de Salamanca / CC BY-NC-ND

Siglo Cero, vol. 49 (1), n. ${ }^{\circ}$ 265, 2018, enero-marzo, pp. 7-19 


\begin{tabular}{|l|l|}
\hline \multicolumn{2}{|c|}{ TABLA 6. Alineación de las habilidades de pensamiento crítico y las estrategias } \\
de transformación (cont.)
\end{tabular}

\section{Conclusión}

En resumen, vivimos tiempos de cambio. El cambio se ve impulsado, en parte, por recursos menguantes, una creciente necesidad de servicios y apoyos, y factores sociopolíticos en constante cambio. La buena noticia es que el campo de las DID se está adaptando a estos cambios, retos e ideas que hemos presentado en este artículo. Cada vez más, los legisladores y los proveedores de servicios y apoyos reconocen la influencia sobre las personas de diferentes sistemas, el enfoque de la discapacidad se está volviendo holístico, las políticas sobre discapacidad se elaboran de una forma sistemática, los apoyos abarcan los diferentes elementos de un sistema de apoyos, se comprenden mejor los puntos de interés de la evaluación, toma auge la importancia de la toma de decisiones basada en evidencias y se produce la transformación de las organizaciones. El mayor reto tanto para los legisladores como para los profesionales reside en seguir evolucionando y convirtiendo esos retos en políticas y prácticas que mejoren las vidas de las personas creando entornos que impulsen el crecimiento y el desarrollo, que apoyen a las personas y que se adapten a las necesidades psicológicas de los individuos y sus familias para lograr la autonomía, la competencia y el establecimiento de nexos personales.

\section{Referencias bibliográficas}

Claes, C., Ferket, N., Vandevelde, S., Verlet, D. y De Maeyer, J. (2017). Disability policy evaluation: Combining logic models and systems thinking. Intellectual and Developmental Disabilities, 55, 247-257.

Claes, C., van Hove, G., Vandevelde, S., van Loon, J. y Schalock, R. L. (2012). The influence of support strategies, environmental factors, and client characteristics on quality of life-related outcomes. Research in Developmental Disabilities, 33, 96-103.

Gómez, L. E. y Verdugo, M. Á. (2016). Outcomes evaluation. En R. L. Schalock y K. D. KeITH (Eds.), Cross-cultural quality of life: Enhancing the lives of people with intellectual disability (2nd ed.) (pp. 71-80). Washington, DC: American Association on Intellectual and Developmental Disabilities.

Lombardi, M., Chiu, C-Y., Schalock, R. L. y Claes, C. (2017). Aligning unCrpd article, quality of life domains, and supports: International Delphi Study. Gent, Belgium: University College Gent. 


\section{SEIS IDEAS QUE ESTÁN CAMBIANDO EL CAMPO DE LAS DISCAPACIDADES INTELECTUALES \\ Y DEL DESARROLLO EN TODO EL MUNDO \\ ROBERT L. SCHALOCK}

Nicholls, J. (2017). Social return on investment-Development and convergence. Evaluation and Program Planning, 64, 127-135.

ReINDERs, H. (2008). The transformation of human services. Journal of Intellectual Disability Research, 52, 564-571.

SCHALOCK, R. L. (2017). Introduction to the special issue on disability policy in a time of change. Intellectual and Developmental Disabilities, 55, 215-222.

Schalock, R. L., Gómez, L. E., Verdugo, M. Á. y Claes, C. (2017). Evidence and evidencebased practices: Are we there yet? Intellectual and Developmental Disabilities, 55, 112-119.

Schalock, R. L. y Keith, K. D. (2016). Setting the cross-cultural agenda of quality of life to enhance the lives of people with intellectual disability. En R. L. Schalock y K. D. Keith (Eds.), Cross-cultural quality of life: Enhancing the lives of people with intellectual disability (2 ${ }^{\text {nd }}$ ed.) (pp. 71-80). Washington, DC: American Association on Intellectual and Developmental Disabilities.

Schalock, R. L., Luckasson, R., Tasse, M. J. y Verdugo, M. Á. (en prensa). A holistic theoretical approach to intellectual disability: Going beyond the four current perspectives. Intellectual and Developmental Disabilities.

SCHAlOCK, R. L. y Verdugo, M. Á. (2012). A leadership guide for today's disabilities organizations: Overcoming challenges and making change happen. Baltimore: Brookes.

SChalock, R. L. y Verdugo, M. Á. (2013). The transformation of disabilities organizations. Intellectual and Developmental Disabilities, 51, 273-286.

Schalock, R. L., Verdugo, M. Á. y Gómez, L. E. (2011). Evidence-based practices in the field of intellectual and developmental disabilities. Evaluation and Program Planning, 34, 273-282.

Schalock, R. L., Verdugo, M. Á. y Lee, T. (2016). A systematic approach to an organization's sustainability. Evaluation and Program Planning, 56, 56-63.

Schalock, R. L., Verdugo, M. Á. y van Loon, J. (enviado para publicación). Understanding organization transformation in evaluation and program planning.

Shogren, K. A., Luckasson, R. y Schalock, R. L. (2015). Using context as an integrative framework to align policy goals, supports, and outcomes in intellectual disability. Intellectual and Developmental Disabilities, 53, 367-376.

Turnbull, R. y Stowe, M. (2017). A model for analyzing disability policy. Intellectual and Developmental Disabilities, 55, 223-233.

Verdugo, M. Á., Jenaro, C., Calvo, I. y Navas, P. (2017). Disability policy implementation from a cross-cultural perspective. Intellectual and Developmental Disabilities, 55, 234-246.

Verdugo, M. Á., Navas, P., Gómez, L. E. y Schalock, R. L. (2012). The concept of quality of life and its role in enhancing human rights in the field of intellectual disability. Journal of Intellectual Disability Research, 56, 1036-1045.

YATES, B. T. y MARRA, M. (2017). Introduction: Social return on investment (SROI). Evaluation and Program Planning, 64, 95-97. 